US Army Corps

of Engineers $S_{\circledast}$

Engineer Research and

Development Center

\title{
Evaluating Parametric Probability Density Functions for Urban Acoustic Noise
}

Matthew J. Kamrath, D. Keith Wilson, Carl R. Hart, Daniel J. Breton,

August 2020 and Caitlin E. Haedrich 
The U.S. Army Engineer Research and Development Center (ERDC) solves the nation's toughest engineering and environmental challenges. ERDC develops innovative solutions in civil and military engineering, geospatial sciences, water resources, and environmental sciences for the Army, the Department of Defense, civilian agencies, and our nation's public good. Find out more at www.erdc.usace.army.mil.

To search for other technical reports published by ERDC, visit the ERDC online library at https://erdclibrary.on.worldcat.org/discovery. 


\section{Evaluating Parametric Probability Density Functions for Urban Acoustic Noise}

Matthew J. Kamrath, D. Keith Wilson, Carl R. Hart, Daniel J. Breton, and Caitlin E. Haedrich

Cold Regions Research and Engineering Laboratory

U.S. Army Engineer Research and Development Center

72 Lyme Road

Hanover, NH 03712

Final report

Approved for public release; distribution is unlimited.

Prepared for U.S. Army Corps of Engineers

Washington, DC 20314

Under Program Element 0611102T, Project Number T442, and Task Number 01 


\section{Preface}

This study was conducted for the U.S. Army Corps of Engineers under Program Element 0611102T, Project T442, and Task Number 01. The Technical monitor was Dr. Marino A. Niccolai.

The work was performed by the Signature Physics Branch of the Research and Engineering Division, U.S. Army Engineer Research and Development Center, Cold Regions Research and Engineering Laboratory (ERDCCRREL). At the time of publication, Mr. Dr. Marino A. Niccolai was Branch Chief; Mr. James Horne was Division Chief; and Dr. Robert Davis was the Technical Director for Geospatial Research and Engineering. The Deputy Director of ERDC-CRREL was Mr. David B. Ringelberg, and the Director was Dr. Joseph L. Corriveau.

This paper was originally published as a proceeding of Internoise 2019, the 48th International Congress and Exhibition on Noise Control Engineering held June 16-19, 2019, in Madrid, Spain.

The Commander of ERDC was COL Teresa A. Schlosser and the Director was Dr. David W. Pittman. 


\title{
Evaluating parametric probability density functions for urban acoustic noise
}

\begin{abstract}
This paper evaluates the suitability of three parametric probability density functions for characterizing urban acoustic noise. For that purpose, the sound levels in onethird-octave bands $(6.3 \mathrm{~Hz}-20 \mathrm{kHz})$ were measured every 0.5 seconds for 5 minutes (for a total of 600 measurements) at 38 locations in Boston, USA. The probability density functions for this dataset were approximated using histograms and the log-normal, generalized gamma, and compound gamma distributions. Maximizing the log-likelihood for each distribution yielded their parameters. The suitability of each distribution was evaluated using the Kullback-Leibler divergence with the histogram approximation as the reference. Overall, the compound gamma distribution was the most accurate followed by the log-normal and then the generalized gamma distributions. Nonetheless, the simplicity of the two-parameter lognormal distribution might be preferred over the three-parameter compound gamma distribution in some applications. For the compound gamma distribution, the distributions of its parameters across all locations and frequencies were also approximated parametrically, which provided satisfactory agreement.
\end{abstract}

\section{INTRODUCTION}

Noise characterization facilitates noise control by providing information about the sources (steady or intermittent) and transmission paths (strong or weak scattering). This paper characterizes urban acoustic noise measurements using parametric probability density functions (PDFs). Previous authors ${ }^{1,2}$ have measured urban acoustic noise but have not rigorously identified an appropriate PDF for their data. García and Faus ${ }^{1}$ note that at very noisy locations the distribution of the levels is nearly Gaussian, but at relatively quiet locations it strays from a Gaussian distribution and has greater skewness. Song and Lenchine ${ }^{2}$ also report some non-Gaussian distributions, but neither paper identifies an appropriate PDF. Ewart and Percival ${ }^{3}$ proposed using the generalized gamma 
distribution to model ocean acoustic intensity measurements because it can represent exponential distributions exactly and can approximate log-normal distributions, which correspond to strong and weak scattering. A log-normal distribution of intensities is equivalent to a Gaussian distribution of intensity levels, which is what García and Faus observed at some of their measurement locations.

While Ewart and Percival demonstrated that the generalized gamma distribution works well for modeling scattered, transmitted signals in the ocean, this distribution does not work as well for background, urban noise. For example, Figure 1 illustrates how the log-normal and generalized gamma distributions approximate García and Faus's data at loud and quiet locations using the maximum log-likelihood parameter estimates. In addition, Table 1 gives the Kullback-Leibler (KL) divergence ${ }^{4}$ for both distributions. The KL divergence quantifies the difference between two PDFs, so smaller values indicate stronger similarity between PDFs. The original levels have been transformed to squared pressures and normalized by the mean squared pressure. Unfortunately, since the original data was A-weighted, these are not the actual squared pressures. Figure 1 and Table 1 demonstrate that for these two cases the two-parameter log-normal distribution better represents the data than the three-parameter generalized gamma distribution.
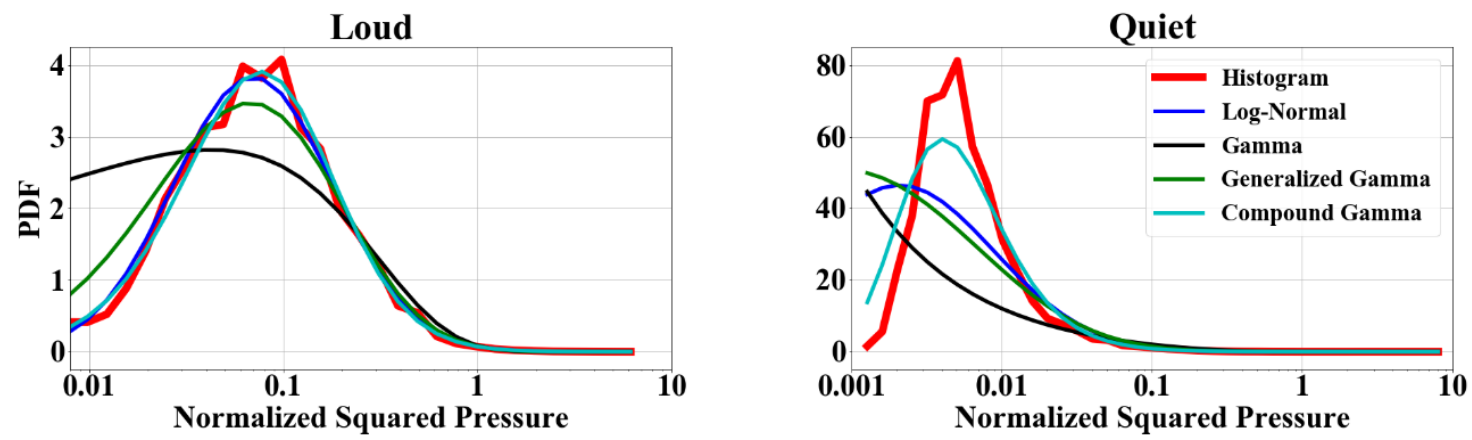

Figure 1. The approximate PDFs for Garcia and Faus's data ${ }^{1}$ with four parametric distributions. The compound gamma distribution characterizes the data the best.

Table 1. The KL divergence for two cases in Garcia and Faus's data ${ }^{1}$ using four parametric distributions. With the smallest KL divergences, the compound gamma distribution performs the best in both cases.

\begin{tabular}{lll}
\hline \multirow{2}{*}{ Distribution } & \multicolumn{2}{c}{ KL Divergence } \\
\cline { 2 - 3 } Log-Normal & Loud Site & Quiet Site \\
Gamma & 0.0051 & 0.43 \\
Generalized Gamma & 0.035 & 0.78 \\
Compound Gamma & 0.0041 & 0.10 \\
\hline
\end{tabular}

Wilson et al. ${ }^{5}$ analyze several alternative distributions for modeling acoustic scattering and suggest that these distributions may be appropriate for modeling urban acoustic noise. In particular, they identify the compound gamma distribution ${ }^{6}$ as a theoretically attractive model because it is derived from a well-defined set of assumptions and allows Bayesian sequential updating. They derive the compound gamma distribution using a gamma distribution for the local model and allowing the gamma distribution's rate parameter to vary according to a second gamma distribution across space, time, and frequency. Naively assuming that García and Faus's data is a measurement of the local 
model (i.e. a gamma distribution) produces a poor approximation of the data, as Figure 1 and Table 1 demonstrate. Instead, since the data is collected across several minutes of time, the data is actually considered a measurement of the global model (i.e. a compound gamma distribution) that has been averaged across only a short period of time and at one location and one frequency, that is a quasi-local model. In addition, Figure 1 and Table 1 provide some practical motivation for choosing a compound gamma distribution by demonstrating that it approximates García and Faus's data better than the other distributions.

This paper assesses whether the log-normal, generalized gamma, and compound gamma distributions are appropriate models for urban acoustic noise based on a one-day measurement campaign in the North End of Boston, USA (Figure 2). It describes in detail three locations with low, medium, and high noise levels and gives summary statistics for the parameters of the compound gamma distribution.



Figure 2. Measurement locations in the North End (Boston, USA). A 5-minute measurement was recorded at each blue flag. Image created using Google Earth Pro. 


\section{PROCEDURE}

On June $7^{\text {th }}, 2018$, the sound level was measured at 38 locations in the North End (Figure 2) of Boston, USA. At this site, road traffic is the dominant sound source with additional contributions from construction sites, pedestrians, air conditioners, and Boston Logan International Airport.

Each measurement consisted of a 5-minute stationary measurement using a Norsonic Sound Analyzer (Nor 140) where the microphone was $1.25 \pm 0.05 \mathrm{~m}$ from the ground (Figure 3 ). Using the fast response setting (125 ms time constant), the sound level meter recorded one measurement every $0.5 \mathrm{~s}$ at the $6.3 \mathrm{~Hz}$ to $20 \mathrm{kHz}$ one-third-octave bands. Each measurement site was also documented with a picture, the time, its GPS coordinates, and a subjective assessment of the dominant sound sources (Table 2).

High: 114

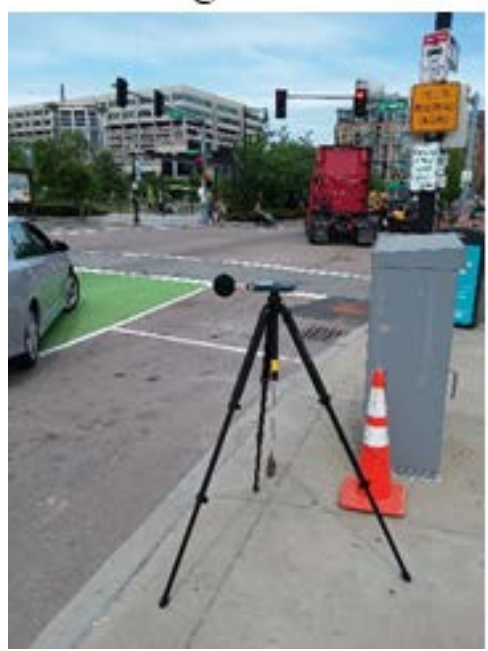

Medium: 127

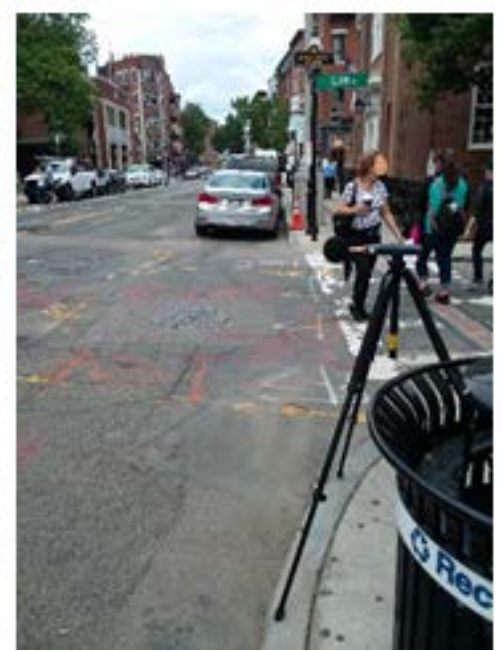

Low: 131

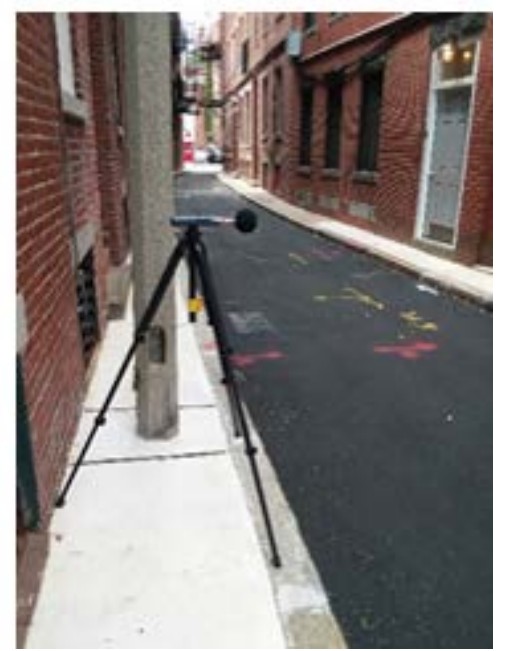

Figure 3. Pictures of three measurement sites representing high, medium, and low sound level areas. The numbers give the corresponding GPS waypoints from Figure 2.

Table 2. Documentation of three measurement sites.

\begin{tabular}{|c|c|c|c|c|c|}
\hline Site & $\begin{array}{l}\text { Time } \\
\text { (EDT) }\end{array}$ & $\begin{array}{l}\text { Cross } \\
\text { Streets }\end{array}$ & $\begin{array}{l}\text { GPS } \\
\text { Coordinates }\end{array}$ & $\begin{array}{l}\text { Noise } \\
\text { Level }\end{array}$ & Description \\
\hline 114 & $10: 30$ & $\begin{array}{l}\text { Cross St } \\
\text { Hanover St }\end{array}$ & $\begin{array}{l}42.362492 \\
-71.055568\end{array}$ & High & $\begin{array}{l}\frac{\text { Very busy street }}{\text { sirens }(10: 31) ; \text { truck idling }} \\
\text { distant generator at } 63 \mathrm{~Hz}\end{array}$ \\
\hline 127 & $13: 30$ & $\begin{array}{l}\text { Hanover St } \\
\text { Clark St }\end{array}$ & $\begin{array}{l}42.365222 \\
-71.053003\end{array}$ & Medium & $\begin{array}{l}\text { Semi-busy street } \\
\text { road/engine noise } \\
\text { pedestrian noise }\end{array}$ \\
\hline 131 & $14: 10$ & $\begin{array}{l}\text { Hanover } \\
\text { Ave }\end{array}$ & $\begin{array}{l}42.365734 \\
-71.052181\end{array}$ & Low & $\begin{array}{l}\text { Quiet side street } \\
\text { birds; air conditioner } \\
\text { distant pedestrians }\end{array}$ \\
\hline
\end{tabular}




\section{RESULTS}

\subsection{Site descriptions}

For simplicity, this paper focuses on 3 of the 38 locations, and Figure 4 gives their spectrograms. The first location (waypoint 114) had very high sound levels due to nearby road traffic and a nearby construction site (especially at $63 \mathrm{~Hz}$ ). A police car with its sirens on $(2 \mathrm{kHz})$ passed about one minute into the measurement. In addition, a large truck stopped near the measurement location while waiting at a red light (minutes 3-4). The second location (waypoint 127) had moderate sound levels from predominately road traffic and pedestrians. The spectrogram demonstrates the intermittent nature of the traffic noise. The third location (waypoint 131) had low sound levels primarily from an air conditioner $(250-315 \mathrm{~Hz})$ and birds $(4-5 \mathrm{kHz})$. These three locations were chosen to give both an idea of what is typical of this neighborhood (medium) and the great variance within this neighborhood (high and low).
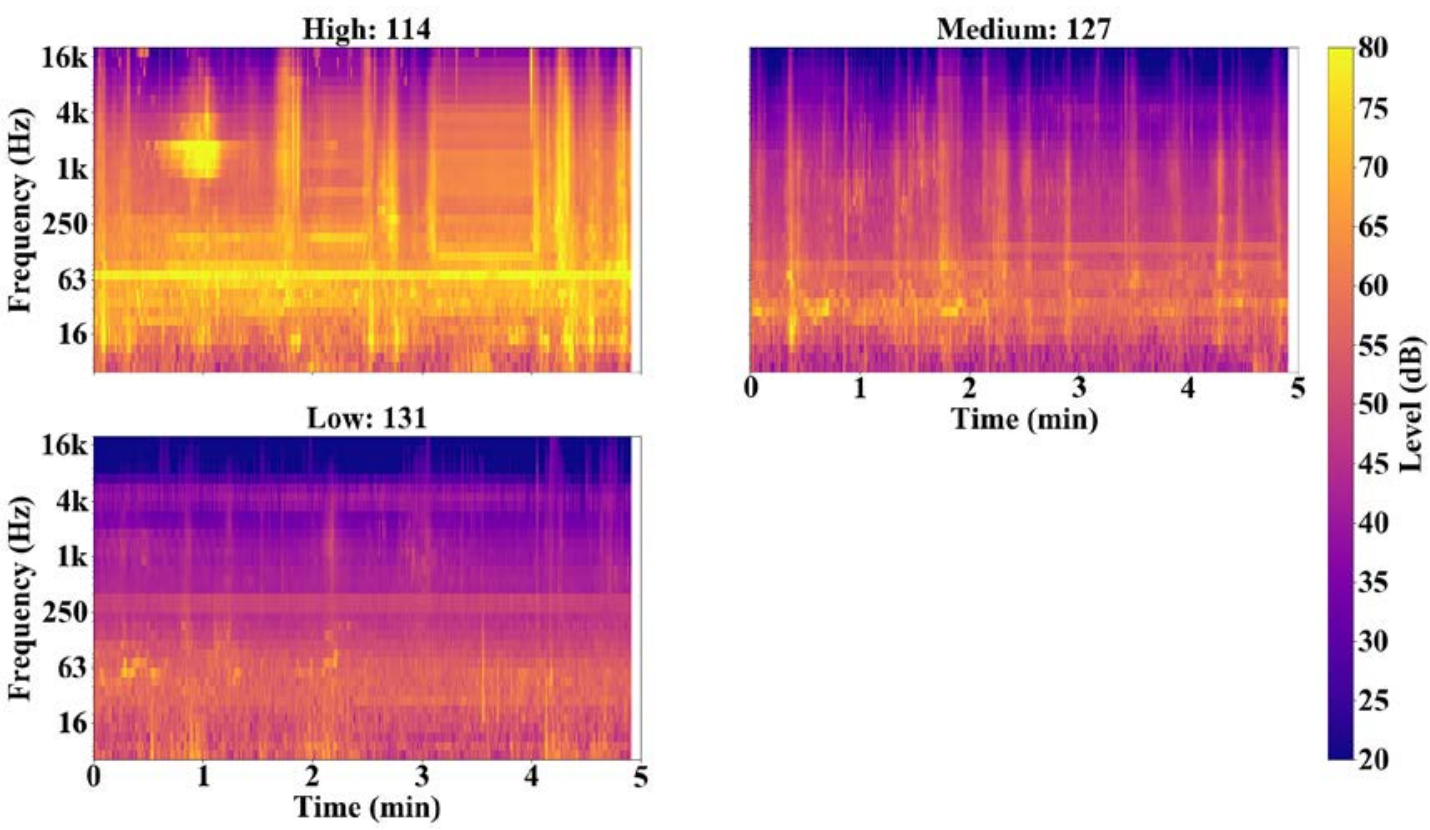

Figure 4. Spectrograms of the sound level meter data at three locations, which represent high, medium, and low sound level areas.

\subsection{Probability Density Functions}

After performing the measurements, the next step was to approximate the PDFs. First, all of the data was transformed from an unweighted (Z-weighting) level $L_{i}$ to a normalized squared pressure $p_{n, i}^{2}$ :

$$
\begin{gathered}
p_{i}^{2} \propto 10 \frac{L_{i}}{10}, \\
\mu_{p^{2}}=\frac{1}{N} \sum_{i=1}^{N} p_{i}^{2}, \\
p_{n, i}^{2}=\frac{p_{i}^{2}}{\mu_{p^{2}}} .
\end{gathered}
$$


The proportionality constant in Equation (1) is in both the numerator and denominator of Equation (3), so it cancels out. The $n$ indicates that the pressure is normalized, and the $i$ indicates that it is an individual pressure measurement. The pressure measurements vary across space, time, and frequency. For this dataset, the PDF is approximated at each location and frequency, averaging across time in Equation (2), using almost 600 pressure measurements within a 5-minute time window.

Histograms and kernel density estimation are non-parametric methods to approximate PDFs. This project used histograms for their transparency, which had 18 logarithmically-spaced bins from 0.01 to 10 (dimensionless squared pressure). The same bins can be used for all of the data because each 5-minute dataset has been normalized by its own mean. The top row of Figure 5 illustrates the PDFs at three locations as a function of the normalized squared pressure and frequency. These plots are made by creating a histogram for each one-third-octave frequency band, so each row may have a different normalization constant, $\mu_{p^{2}}$.

The histograms in Figure 5 demonstrate several characteristics of urban acoustic noise. First, each one-third-octave frequency band (i.e. each row) is very similar to its adjacent frequency bands (i.e. the rows above and below it). This result suggests that most of the urban sound sources are broadband sources. The most notable exception is in the high noise level plot (site 114) at $2 \mathrm{kHz}$ because a police siren, which is not broadband, sounded during part of the measurement. Moreover, there is a single well-defined peak for each frequency, which creates a sideways U-shaped ridge in these plots. The bottom of the $U$ is at the mid-range frequencies (about $250 \mathrm{~Hz}$ ) with the tops at the low and high frequencies $(12.5 \mathrm{~Hz}$ and $12.5 \mathrm{kHz})$. This shape indicates that middle frequencies have a much smaller probability density of having pressures much less than their mean, which creates a valley of probability density on the left side of each plot. Moreover, as the overall level decreases, the valley tends to grow.

The highest plotted probability density occurs at waypoint 114 with the normalized squared pressure equal to 0.01 and the frequency equal to $16 \mathrm{kHz}$. In this case, rare high amplitude events push the mean far above the typical value (i.e. high skewness). The police siren at $2 \mathrm{kHz}$ in plot 114 is a simple example. The siren's presence is rare (only 20 seconds out of a 5 minute measurement), but it has such a high amplitude that it dramatically increases the mean at that frequency.

PDFs can also be approximated using a parametric distribution. This paper evaluates three different distributions: the log-normal, generalized gamma, and compound gamma distributions:

$$
\begin{gathered}
\operatorname{LogNorm}(x \mid \mu, \sigma)=\frac{1}{x \sigma \sqrt{2 \pi}} e^{-\frac{[\ln (x)-\mu]^{2}}{2 \sigma^{2}}}, \\
\operatorname{GenGamma}(x \mid k, \lambda, b)=\frac{b \lambda^{b k} x^{b k-1}}{\Gamma(k)} e^{-(\lambda x)^{b}}, \\
\operatorname{CompGamma}(x \mid a, b, q)=\frac{1}{\mathrm{~B}(a, b)} \frac{(x / q)^{a-1}}{q(1+x / q)^{a+b}},
\end{gathered}
$$

where $\Gamma$ is the gamma function, $B$ is the beta function, $x$ is the random variable, and the remaining letters are the distribution parameters, which can be determined by using the log-likelihood estimates. The second, third, and fourth rows of Figure 5 respectively illustrate the log-normal, generalized gamma, and compound gamma approximations at 
three different locations. The distribution parameters are calculated for each one-third-octave frequency band and location.
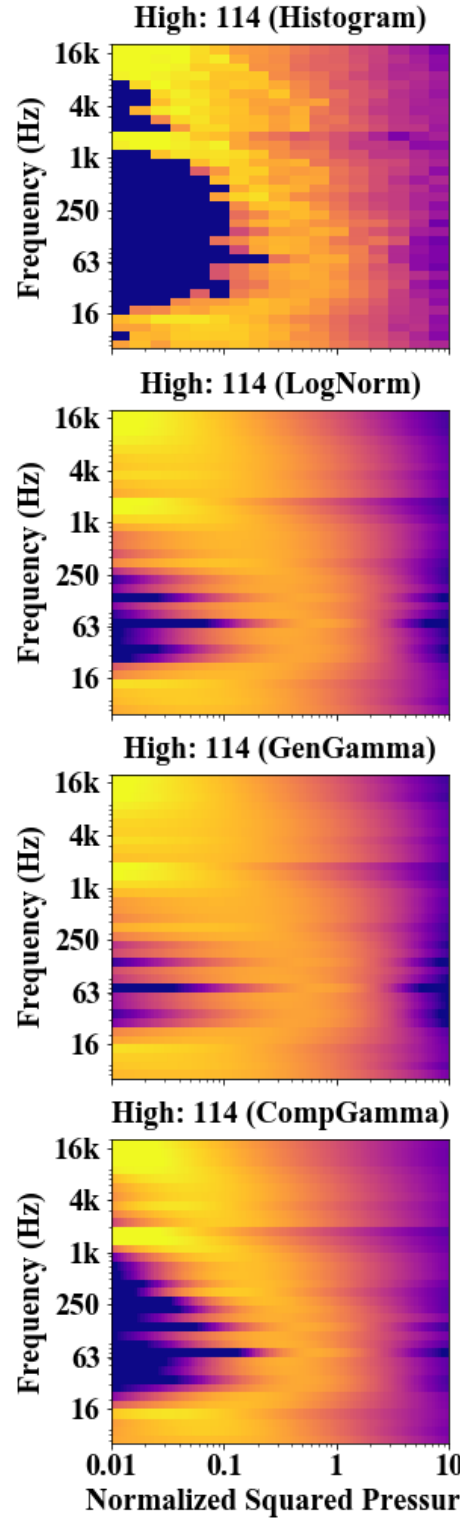
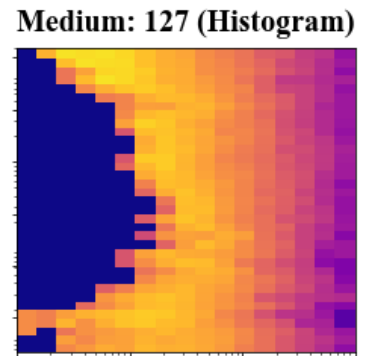

Medium: 127 (LogNorm)

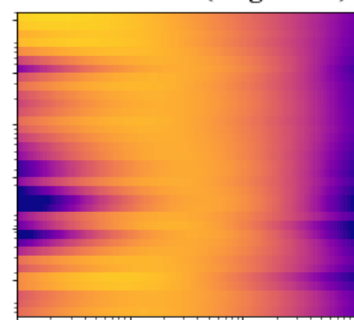

Medium: 127 (GenGamma)

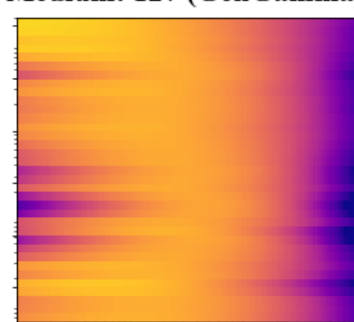

Medium: 127 (CompGamma)

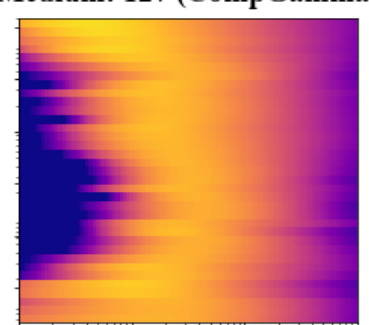

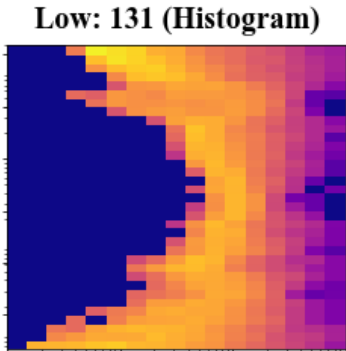

Low: 131 (LogNorm)

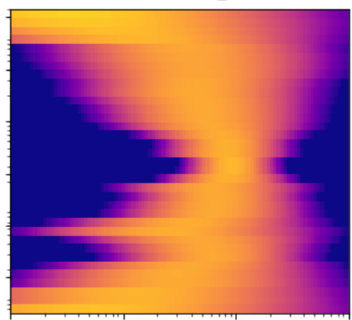

Low: 131 (GenGamma)

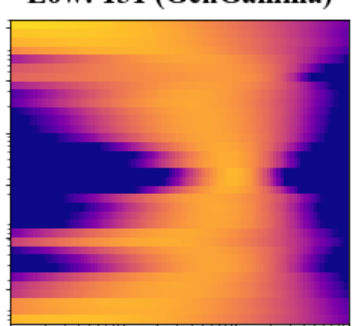

Low: 131 (CompGamma)

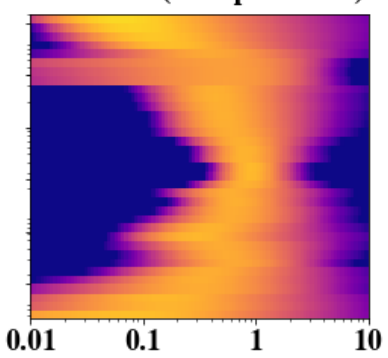

$-0.001$

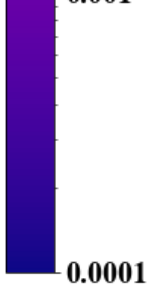

Figure 5. The PDFs at three locations estimated using a histogram and three parametric distributions. The compound gamma distribution is the most accurate.

The compound gamma distribution is qualitatively the best parametric representation of the histograms, but all three parametric distributions have some features that could be improved. All three systematically over predict the probability density of pressures in the valley; visually, the dark blue areas on the left side of the histograms are reduced in size in the parametric approximations. However, the compound gamma distribution reduces the size of the low probability density valley the least. In addition, each of the parametric distributions has some issues at the low sound level location (site 131) at the high frequencies, specifically, above $8 \mathrm{kHz}$ for the log-normal and generalized gamma distributions and at $3-8 \mathrm{kHz}$ for the compound gamma distribution.

For more detail about the actual values, Figure 6 plots the PDFs at two frequencies $(250 \mathrm{~Hz}$ and $1 \mathrm{kHz}$ ) and three locations using four different approximations. Using the histograms as a reference, the compound gamma distribution is the most similar. All of the parametric distributions tend to underestimate the height of the peak and overestimate 
the width of the peak, but the compound gamma has the smallest error. In addition, the log-normal and generalized gamma distributions tend to underestimate the location of the peak; whereas, the compound gamma distribution correctly identifies the peak location.

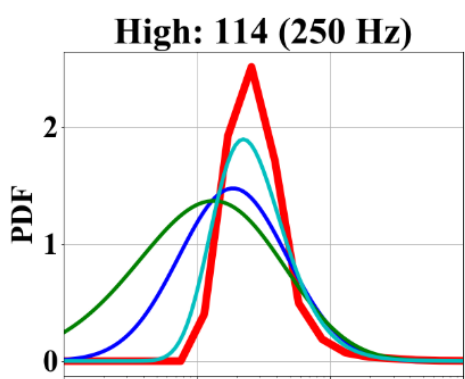

High: $114(1000 \mathrm{~Hz})$

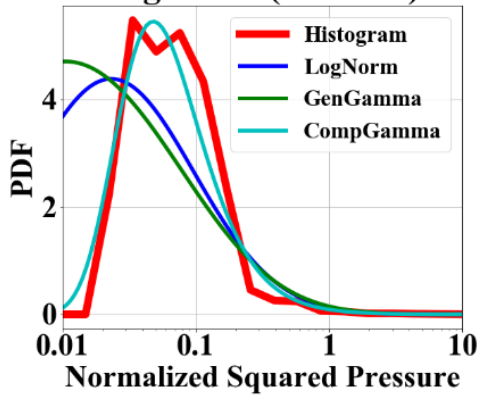

Medium: $127(250 \mathrm{~Hz})$
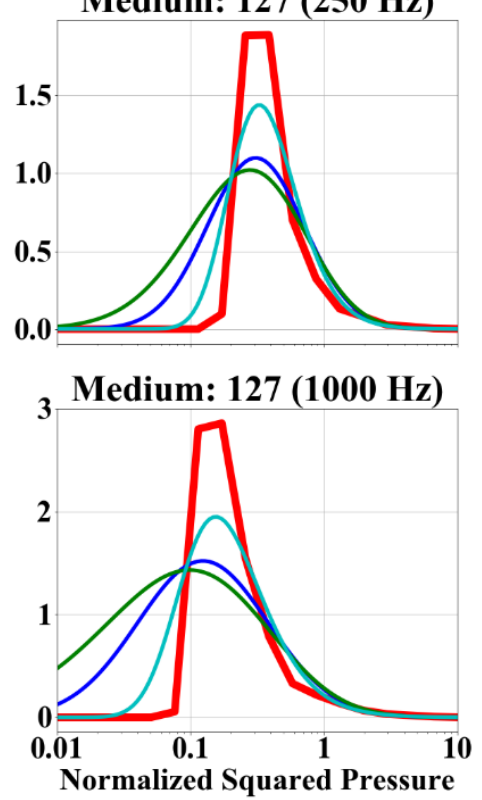

Low: $131(250 \mathrm{~Hz})$

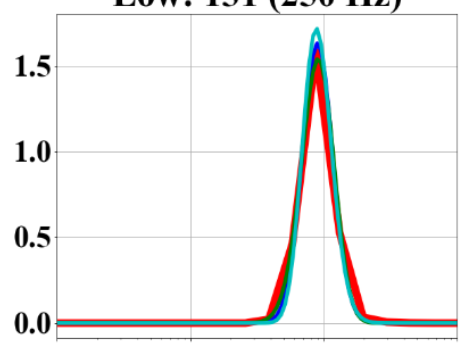

Low: $131(1000 \mathrm{~Hz})$

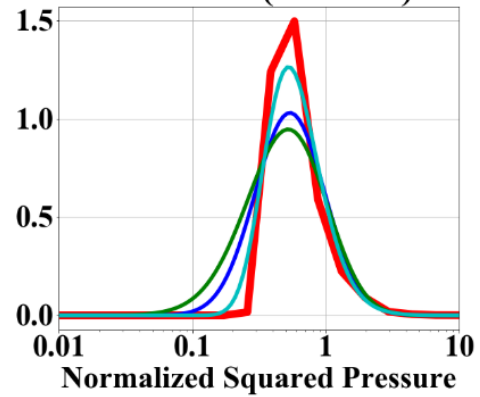

Figure 6. The PDFs at three locations and two frequencies estimated using a histogram and three parametric distributions. The compound gamma distribution is most accurate.

Qualitatively, the compound gamma distribution yields the most accurate parametric approximation. To make these comparisons quantitative and to include all the measurements, Table 3 evaluates the KL divergence across all of the locations and frequencies. Table 3 confirms that the compound gamma distribution approximates urban acoustic noise the best followed by the log-normal and then the generalized gamma distributions. Since the two-parameter log-normal distribution is simpler than the three-parameter compound gamma, the log-normal distribution may be preferred in some applications. However, the three-parameter generalized gamma is more complicated and less accurate than the log-normal distribution. A different parametric PDF may perform better than any of these distributions. Also, additional data (i.e. more than 600 data points for each distribution) could also help by improving the accuracy of the histogram estimates. The samples could be taken more frequently $(>2 \mathrm{~Hz})$ and over a longer time duration ( $>5$ minutes $)^{7}$. In addition, both the visual and KL divergence comparisons are affected by the accuracy of the maximum log-likelihood estimates.

Table 3. KL divergence percentiles across all locations and frequencies for three distributions using the histograms as the reference.

\begin{tabular}{llllllll}
\hline & \multicolumn{7}{c}{ Percentile } \\
\cline { 2 - 8 } Distribution & $1 \%$ & $10 \%$ & $25 \%$ & $50 \%$ & $75 \%$ & $90 \%$ & $99 \%$ \\
\hline Log-Normal & 0.0080 & 0.025 & 0.051 & 0.11 & 0.20 & 0.35 & 0.65 \\
Generalized Gamma & 0.012 & 0.039 & 0.076 & 0.15 & 0.27 & 0.44 & 0.73 \\
Compound Gamma & 0.0038 & 0.011 & 0.021 & 0.043 & 0.091 & 0.18 & 0.40 \\
\hline
\end{tabular}




\subsection{Hyperparameters}

Calculating the compound gamma distribution parameters $(a, b, q)$ at all 38 locations and 36 frequencies enables an analysis of the distribution of those parameters. The parameters $(\mu, \sigma)$ that characterize the distributions of those parameters $(a, b, q)$ are called hyperparameters (i.e. the parameters of different parameters). Figure 7 illustrates two different approximations of the underlying PDF: a histogram with 10 logarithmically-spaced bins and a log-normal distribution. Table 4 gives the hyperparameters for each of the log-normal distributions where each of the compound gamma parameters have been scaled by its sample mean. Table 4 also provides the KL divergence for each distribution. Overall, the log-normal distribution appears to be a good fit for the compound gamma distribution parameters. The biggest deviation is at very small values of $q$.
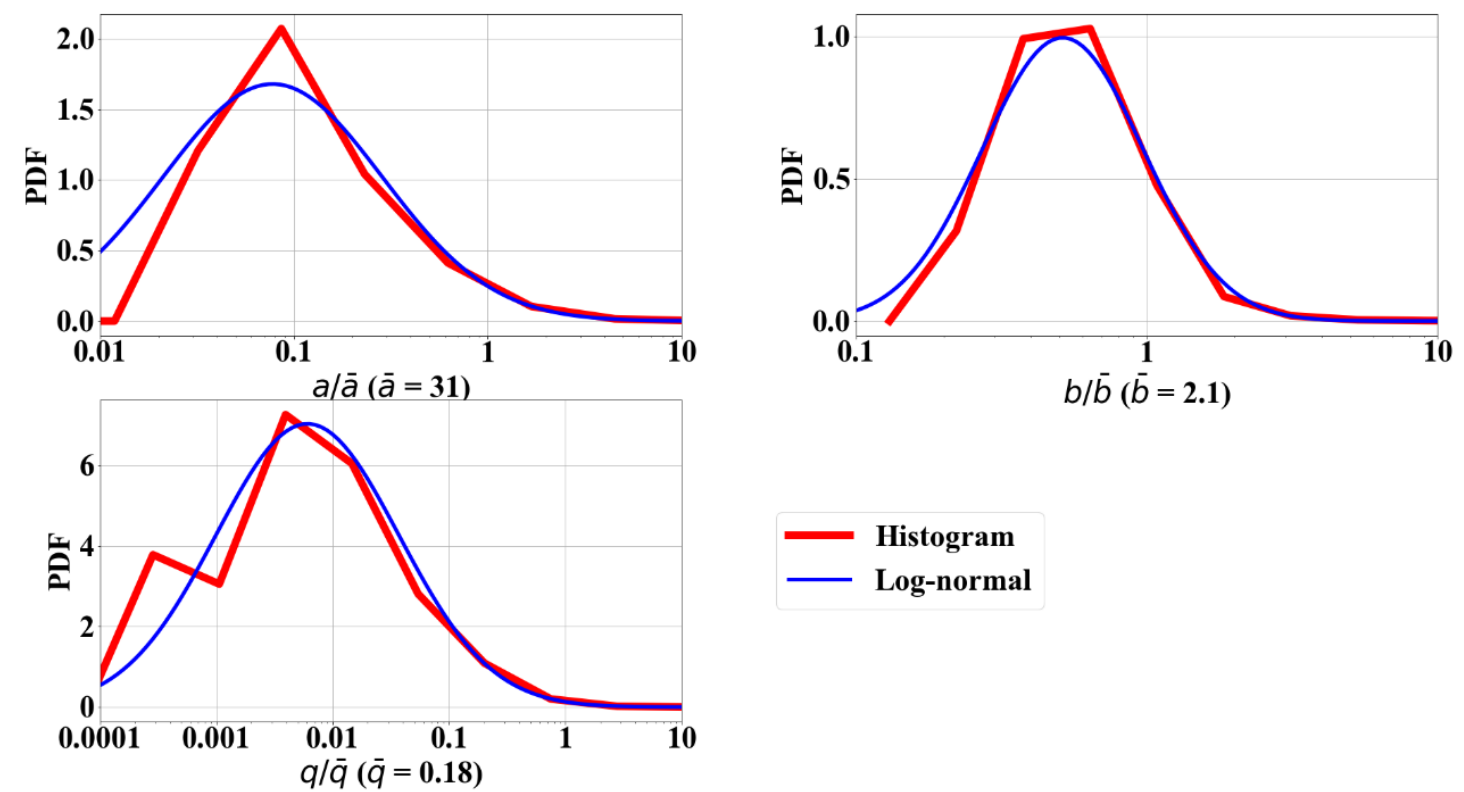

Figure 7. The PDFs of the compound gamma distribution parameters. The log-normal distribution provides a satisfactory approximation of the histograms.

Table 4. Hyperparameters and KL divergence for each compound gamma parameter.

\begin{tabular}{cccc}
\hline \multirow{2}{*}{$\begin{array}{c}\text { Compound Gamma } \\
\text { Parameter }\end{array}$} & \multicolumn{2}{c}{ Log-Normal Hyperparameter } & \\
\cline { 2 - 3 } & $\mu$ & $\sigma$ & KL Divergence \\
\hline$a$ & -0.85 & 1.3 & 0.013 \\
$b$ & -0.26 & 0.64 & 0.013 \\
$q$ & -1.8 & 1.8 & 0.045 \\
\hline
\end{tabular}




\section{CONCLUSION}

An experimental campaign was conducted in Boston, USA to sample urban acoustic noise so that it could be characterized using parametric PDFs. This research provides insight into how well the log-normal, generalized gamma, and compound gamma distributions approximate urban acoustic noise. Evaluating them using KL divergence, the compound gamma distribution performs the best followed by the log-normal and generalized gamma distributions. All of them show some systematic errors (e.g. underestimating the peak height and overestimating the peak width), so another PDF might perform better.

\section{ACKNOWLEDGEMENT}

This research was funded by the Assistant Secretary of the Army (Acquisition, Logistics, and Technology) through the project "Geography of RF and Acoustic Urban Noise". Permission to publish was granted by the Director, Cold Regions Research and Engineering Laboratory. Any opinions expressed in this paper are those of the authors, and are not to be construed as official positions of the funding agency or the Department of the Army unless so designated by other authorized documents.

\section{REFERENCES}

1. García A, Faus LJ. Statistical analysis of noise levels in urban areas. Appl Acoust. 1991;34(4):227-247. doi:10.1016/0003-682X(91)90007-2

2. Song J, Lenchine V V. Statistical properties of urban noise - results of a long term monitoring program. In: Proceedings of Acoustics 2017. Perth, Australia; 2017. https://www.acoustics.asn.au/conference_proceedings/AAS2017/papers/p36.pdf.

3. Ewart TE, Percival DB. Forward scattered waves in random media-The probability distribution of intensity. J Acoust Soc Am. 1986;80(6):1745-1753. doi:10.1121/1.394288

4. Kullback S, Leibler RA. On information and sufficiency. Ann Math Stat. 1951;22(1):79-86. doi:10.1214/aoms/1177729694

5. Wilson DK, Hart CR, Pettit CL, Breton DJ, Nykaza ET, Ostashev VE. Scattered signal distributions, parametric uncertainties, and Bayesian sequential updating. Proc Mtgs Acoust. 2017;31(055002). doi:10.1121/2.0000672

6. DuBey SD. Compound gamma, beta and F distributions. Metrika. 1970;16(1):2731. doi:10.1007/BF02613934

7. Brocolini L, Lavandier C, Quoy M, Ribeiro C. Measurements of acoustic environments for urban soundscapes: Choice of homogeneous periods, optimization of durations, and selection of indicators. J Acoust Soc Am. 2013;134(1):813-821. doi:10.1121/1.4807809 


\section{REPORT DOCUMENTATION PAGE}

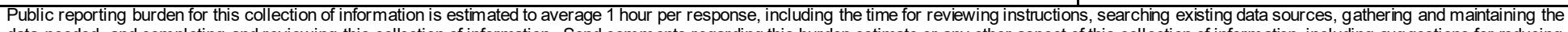

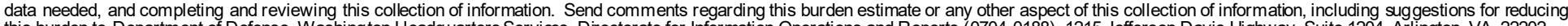

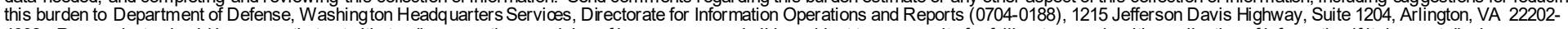

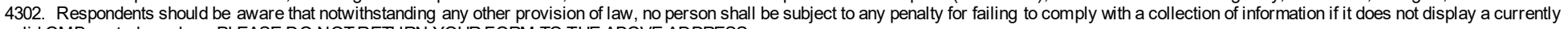
valid OMB control number. PLEASE DO NOT RETURN YOUR FORM TO THE ABOVE ADDRESS.
1. REPORT DATE
August 2020
2. REPORT TYPE

4. TITLE AND SUBTITLE

Final

. DATES COVERED (From - To)

5a. CONTRACT NUMBER

Eva luating Pa rametric Probability Density Functions for Urban Acoustic Noise

5b. GRANT NUMBER

5c. PROGRAM ELEMENT NUMBER

$0611102 \mathrm{~T}$

6. AUTHOR(S)

5d. PROJECT NUMBER

T442

Matthew J. Kamrath, D. Keith Wilson, CarlR. Hart, DanielJ. Breton, and Caitlin E. Haedrich

5e. TASK NUMBER

01

5f. WORK UNIT NUMBER

7. PERFORMING ORGANIZATION NAME(S) AND ADDRESS(ES)

U.S. Army Engineer Research and Development Center

8. PERFORMING ORGANIZATION REPORT NUMBER

Cold Regions Research and Engineering Laboratory

72 Lyme Road

ERDC/CRREL MP-20-15

Hanover, NH 03712 S

9. SPONSORING / MONITORING AGENCY NAME(S) AND ADDRESS(ES)

10. SPONSOR/MONITOR'S ACRONYM(S)

U.S. Army Corps of Engineers

Washington, DC 20314-1000

USACE

11. SPONSOR/MONITOR'S REPORT NUMBER(S)

\section{DISTRIBUTION / AVAILABILITY STATEMENT}

Approved for public release; distribution is unlimited.

\section{SUPPLEMENTARY NOTES}

Origina lly published as a proceeding of INTERNOISE20 19, Madrid, Spain, June 16-19, 2019.

\section{ABSTRACT}

This paper evaluates the suitability of three parametric probability density functions for characterizing urban acoustic noise. For that purpose, the sound levels in one-third-octave bands $(6.3 \mathrm{~Hz}-20 \mathrm{kHz}$ ) were measured every 0.5 seconds for 5 minutes (for a total of 600 measurements) at 38 locations in Boston, USA. The probability density functions for this da taset were approximated using histograms and the log-normal, generalized gamma, and compound ga mma distributions. Maximizing the log-likelihood for each distribution yielded their pa rameters. The suitability of each distribution was evaluated using the Kullback-Leibler divergence with the histogram approximation as the reference. Overall, the compound gamma distribution wa s themost accurate followed by the log-normal and then the genera lized ga mma distributions. Nonetheless, the simplicity of the two-parameter log-normal distribution might be preferred over the three-parameter compound the distributions of its pa rameters a cross all loca tions and frequencies were also approximated parametrically, which provided satisfactory a greement.

\section{SUBJECT TERMS}

Urban acoustic noise, probability density function

\begin{tabular}{|c|c|c|c|c|c|}
\hline \multicolumn{3}{|c|}{ 16. SECURITY CLASSIFICATION OF: } & 17. LIMITATION & 18. NUMBER & 19a. NAME OF RESPONSIBLE PERSON \\
\hline $\begin{array}{l}\text { a. REPORT } \\
\text { Unclassified }\end{array}$ & $\begin{array}{l}\text { b. ABSTRACT } \\
\text { Uncla ssified }\end{array}$ & $\begin{array}{l}\text { c. THIS PAGE } \\
\text { Unclassified }\end{array}$ & UU & 13 & $\begin{array}{l}\text { 19b. TELEPHONE NUMBER } \\
\text { (include area code) }\end{array}$ \\
\hline
\end{tabular}

